\title{
Widespread increases in malondialdehyde immunoreactivity in dopamine-rich and dopamine-poor regions of rat brain following multiple, high doses of methamphetamine
}

\author{
Kristen A. Horner ${ }^{1 *}$, Yamiece E. Gilbert ${ }^{1,2}$ and Susan D. Cline ${ }^{1}$ \\ Division of Basic Medical Sciences, Mercer University School of Medicine, Macon, GA, USA \\ 2 Master of Public Health Program, Department of Community Medicine, Mercer University School of Medicine, Macon, GA, USA
}

\section{Edited by:}

Elizabeth Abercrombie, Rutgers-

Newark: The State University of New

Jersey, USA

\section{Reviewed by:}

Christine E. Collins, Vanderbilt

University, USA

Preston E. Garraghty, Indiana

University, USA

\section{*Correspondence:}

Kristen A. Horner, Division of Basic Medical Sciences, Mercer University School of Medicine, 1550 College

Street, Macon, GA 31207, USA. e-mail: horner_ka@mercer.edu
Treatment with multiple high doses of methamphetamine (METH) can induce oxidative damage, including dopamine (DA)-mediated reactive oxygen species (ROS) formation, which may contribute to the neurotoxic damage of monoamine neurons and long-term depletion of DA in the caudate putamen (CPu) and substantia nigra pars compacta (SNpc). Malondialdehyde (MDA), a product of lipid peroxidation by ROS, is commonly used as a marker of oxidative damage and treatment with multiple high doses of METH increases MDA reactivity in the CPu of humans and experimental animals. Recent data indicate that MDA itself may contribute to the destruction of DA neurons, as MDA causes the accumulation of toxic intermediates of DA metabolism via its chemical modification of the enzymes necessary for the breakdown of DA. However, it has been shown that in human METH abusers there is also increased MDA reactivity in the frontal cortex, which receives relatively fewer DA afferents than the $\mathrm{CPu}$. These data suggest that METH may induce neuronal damage regardless of the regional density of DA or origin of DA input. The goal of the current study was to examine the modification of proteins by MDA in the DA-rich nigrostriatal and mesoaccumbal systems, as well as the less DA-dense cortex and hippocampus following a neurotoxic regimen of METH treatment. Animals were treated with METH (10 mg/ $\mathrm{kg}$ ) every $2 \mathrm{~h}$ for $6 \mathrm{~h}$, sacrificed 1 week later, and examined using immunocytochemistry for changes in MDA-adducted proteins. Multiple, high doses of METH significantly increased MDA immunoreactivity (MDA-ir) in the CPu, SNpc, cortex, and hippocampus. Multiple METH administration also increased MDA-ir in the ventral tegmental area and nucleus accumbens. Our data indicate that multiple METH treatment can induce persistent and widespread neuronal damage that may not necessarily be limited to the nigrostriatal DA system.

Keywords: neurotoxicity, lipid peroxidation, striatum, substantia nigra, psychostimulant, dopamine

\section{INTRODUCTION}

Methamphetamine (METH) is a widely abused psychostimulant whose use has dire social and medical consequences. Over 60 million people world-wide report abusing amphetamine-type stimulants, especially METH (Maxwell, 2005). In the United States, METH use is endemic in the Western states, while the epidemic of METH use and abuse is growing notably in the Midwestern and Southern states (Substance Abuse and Mental Health Services Administration, Office of Applied Studies, 2010). The short-term effects of METH use include euphoria, increased locomotor activity, and hyperthermia, whereas long-term use can lead to dependence, addiction, paranoia, and psychosis. Repeated high doses of METH can lead to neurotoxic insults within the basal ganglia of both rodents and humans, which may underlie some of the symptoms of long-term abuse (Hotchkiss and Gibb, 1980; Volkow et al., 2001; Johnson-Davis et al., 2002). In particular, treatment with multiple high doses of METH results in damage to dopamine (DA) neurons of the substantia nigra pars compacta $(\mathrm{SNpc})$, which project heavily to the caudate putamen $(\mathrm{CPu})$, while the $\mathrm{DA}$ neurons in the adjacent ventral tegmental area (VTA), which project to the nucleus accumbens (NAc) are less severely affected (Ricaurte et al.,
1980). The damage to the DA neurons in the SNpc is evidenced by long-term losses in several prototypical markers of dopaminergic neurons, including a loss of DA uptake sites, decreased tyrosine hydroxylase (TH) activity and severely reduced DA tissue levels in the CPu (Kogan et al., 1976; Hotchkiss and Gibb, 1980; Ricaurte et al., 1980; Seiden and Ricaurte, 1987).

The exact mechanism(s) that mediate the enhanced and relatively selective neurotoxicity of nigrostriatal neurons in response to repeated METH treatment are not entirely understood. Several studies have identified oxidative stress as a contributing factor in METH-induced neurotoxicity (Yamamoto and Zhu, 1998; Lavoie and Hastings, 1999; Yamamoto and Bankson, 2005; Krasnova and Cadet, 2009). One of the most common markers of oxidative stress is the lipid peroxidation product malondialdehyde (MDA; Aldini et al., 2007). METH can increase the oxidation of DA, resulting in the formation of reactive DA quinones and superoxide radicals within the terminals of dopaminergic neurons (Lavoie and Hastings, 1999; Krasnova and Cadet, 2009). These reactive oxygen species (ROS) can attack polyunsaturated fatty acids, which are enriched in neuronal membranes, resulting in the generation of MDA (Brown and Yamamoto, 2003; Brown et al., 2005; Del Rio et al., 2005; Krasnova 
and Cadet, 2009; Lieberman and Marks, 2009). Accordingly, enhanced levels of MDA or MDA-like reactivity have been found in the brains of METH addicts and METH-treated experimental animals (Acikgoz et al., 1998; Yamamoto and Zhu, 1998; Kim et al., 1999; Kita et al., 2000; Gluck et al., 2001; Fitzmaurice et al., 2006). However, recent data raises the possibility that MDA is not just the result of oxidative stress and cellular damage, but can act on its own to induce neuronal toxicity (Jinsmaa et al., 2009; Long et al., 2009).

For example, MDA is a reactive electrophile that can modify amino groups in proteins, rendering enzymes non-functional (Del Rio et al., 2005; Aldini et al., 2007). MDA inhibits mitochondrial electron transport proteins, which may lead to ROS generation and mitochondrial dysfunction in neurons (Long et al., 2009). Furthermore, it has been shown that MDA can increase levels of toxic intermediates of DA catabolism (Jinsmaa et al., 2009) and repeated treatment with $\mathrm{METH}$ can increase MDA and other reactive aldehydes in the DA-rich nigrostriatal pathway (Yamamoto and Zhu, 1998; Gluck et al., 2001; Fitzmaurice et al., 2006; Horner et al., 2010). However, recent data show that in human abusers of METH, reactive aldehyde levels are also increased in the frontal cortex, a region that has relatively fewer DA afferents from the SNpc and contains lower tissue levels of DA than the CPu (Emson et al., 1977; Scatton et al., 1983; Johnson-Davis et al., 2002; Fitzmaurice et al., 2006). Together, these data suggest that METH can induce significant oxidative damage regardless of the regional density of DA or origin of DA input. Furthermore, it is possible that the formation of MDA-protein adducts could lead to DA-related cellular damage, as well as non-DA-related damage to neurons via direct interactions of MDA with essential proteins and amino acids.

The purpose of the current study was to examine and compare the effect of multiple high doses of METH on the generation of MDA-adducted protein in the DA-rich nigrostriatal and mesoaccumbal systems. We also examined the generation of MDA-adducted proteins by multiple METH treatment in the hippocampus and cortex, two areas that are less intensely innervated by $\mathrm{DA}$ afferents than the $\mathrm{CPu}$ and NAc. While previous studies have examined MDA reactivity following METH administration in experimental animals and in human METH addicts, many of these studies used the thiobarbituric acid reactive substances (TBARS) assay to detect changes in MDA levels (Acikgoz et al., 1998; Jayanthi et al., 1998; Kim et al., 1999; Kita et al., 2000; Gluck et al., 2001; Fitzmaurice et al., 2006). While a standard approach for MDA measurement in body fluids and tissues, the TBARS assay is not specific for MDA and detects free, non-adducted MDA, including MDA that has been released from its adducts with biomolecules as well as unreacted, diffusible MDA that may not impair cellular function (Moore and Roberts, 1998). The current study employed immunocytochemistry with specificity for MDA-adducted proteins, which allowed us to detect MDA-induced neuronal damage, while maintaining anatomical and cellular resolution. We also examined levels of $\mathrm{TH}$ immunoreactivity ( $\mathrm{TH}$-ir) in the $\mathrm{CPu}$ and the NAc, in order to confirm that repeated METH treatment resulted in a severe depletion of DA in the nigrostriatal pathway as compared to the mesoaccumbal pathway. Finally, we determined whether the levels of MDA-adducted protein induced by multiple METH treatment correlated significantly with the severity of TH depletion in the $\mathrm{CPu}$, as compared to the NAc.

\section{MATERIALS AND METHODS ANIMALS}

Male Sprague-Dawley rats (Harlan Laboratories, Indianapolis, IN, USA), weighing 250-350 g were used in all experiments. Rats were housed in groups of four in plastic cages in a temperaturecontrolled room. Rats were on a 14:10 h light/dark cycle and had free access to food and water. All animal care and experimental manipulations were approved by the Institutional Animal Care and Use Committee of Mercer University School of Medicine and were in accordance with the National Institutes of Health Guide for the Care and Use of Laboratory Animals.

\section{PHARMACOLOGICAL PROCEDURES}

$( \pm)$ Methamphetamine hydrochloride was obtained from the National Institute on Drug Abuse (Bethesda, MD, USA). The drug doses were calculated as the free base, diluted in saline, and administered subcutaneously. On the day of the experiment, the rats were weighed, housed in plastic tub cages (eight rats per cage), and transferred to the laboratory. Animals received a total of four injections of either METH (10 mg/kg, s.c.) or saline with a $2 \mathrm{~h}$-interval between each injection. This dosing regimen has been shown to result in significant depletion of DA in the nigrostriatal system (Hotchkiss and Gibb, 1980; Johnson-Davis et al., 2002).

\section{TISSUE SECTIONING}

One week after exposure to multiple doses of METH, rats were sacrificed by exposure to $\mathrm{CO}_{2}$, followed by decapitation. The brains were rapidly harvested, quick frozen in isopentane on dry ice and stored at $-80^{\circ} \mathrm{C}$. The brains were then cut into $12-\mu \mathrm{m}$ sections on a cryostat (Minotome Plus, Triangle Biomedical Sciences, Durham, NC, USA). Coronal sections were taken through the anterior cingulate cortex (ACC)/prelimbic cortex (PRL; +4.2 mm anterior to bregma), $\mathrm{CPu} / \mathrm{NAc} /$ primary motor cortex $(\mathrm{M} 1 ;+1.7 \mathrm{~mm}$ anterior to bregma), hippocampus ( $-2.92 \mathrm{~mm}$ posterior to bregma), and SN/VTA ( $-4.92 \mathrm{~mm}$ posterior to bregma; Figure 1). Sections were thaw-mounted onto Superfrost Plus slides (VWR, Radnor, PA, USA) and stored at $-20^{\circ} \mathrm{C}$ until their use in immunocytochemical assays.

\section{TH IMMUNOCYTOCHEMISTRY}

A modified peroxidase anti-peroxidase method was used to detect changes in TH-ir in the CPu and NAc (Sternberger, 1979; JohnsonDavis et al., 2002). Slides were thawed, washed in $0.1 \mathrm{M}$ phosphatebuffered saline (PBS) and post-fixed in $4 \%$ paraformaldehyde $/ 0.9 \%$ $\mathrm{NaCl}$ for $10 \mathrm{~min}$. Slides were rinsed three times for $5 \mathrm{~min}$ in PBS and the sections circled with a PAP pen. Non-specific binding was blocked with $10 \%$ normal horse serum/0.3\% Triton-X for $2 \mathrm{~h}$ at room temperature. The blocking solution was then removed, and the sections were incubated overnight in humid chambers at $4^{\circ} \mathrm{C}$ with a monoclonal mouse-anti TH antibody (1:300; Immunostar, Hudson, WI, USA) diluted in $0.3 \%$ Triton-X/PBS. The next day, the slides were rinsed three times for $5 \mathrm{~min}$ in PBS, and incubated for $1.5 \mathrm{~h}$ at room temperature with anti-mouse $(\mathrm{H}+\mathrm{L})$ peroxidase generated in horse (1:150; Vector Laboratories, Burlingame, CA, USA) diluted in PBS/0.3\% Triton-X. The slides were rinsed three times in PBS, and incubated with $0.1 \% \mathrm{DAB} / 0.005 \% \mathrm{H}_{2} \mathrm{O}_{2}$ in PBS for 4-8 min. The slides were rinsed briefly in deionized $\mathrm{H}_{2} \mathrm{O}$, dehydrated in a series of alcohols, and coverslipped out of xylene. 

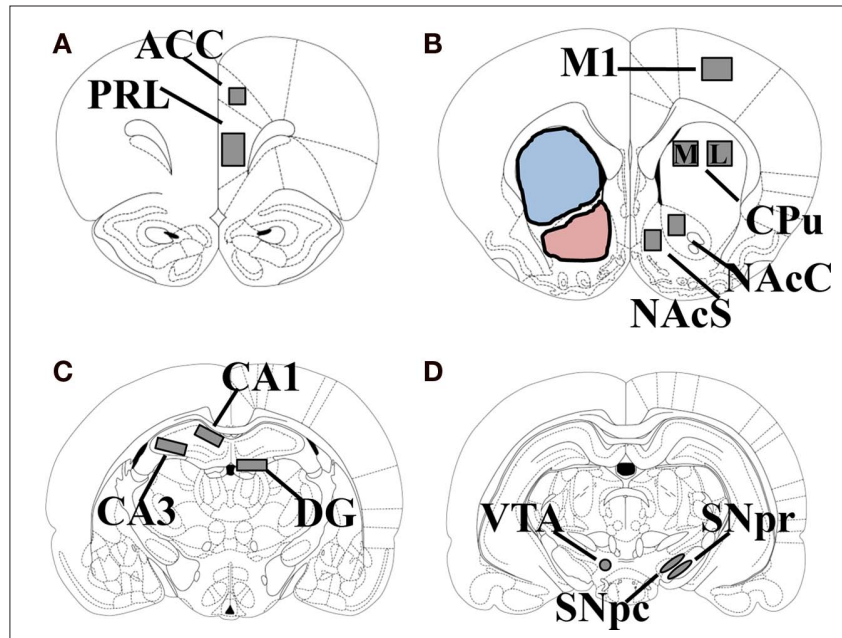

FIGURE 1 | Schematic diagram of the rostral to caudal sections of rat brain used for analysis of malondialdehyde immunoreactivity (MDA-ir) and tyrosine hydroxylase immunoreactivity (TH-ir). Measurements given are relative to bregma. The regions used for analysis of MDA-ir are highlighted in gray and consist of the PRL and ACC (+4.2 mm; $\mathbf{A})$; NAc, CPu, and M1 (+1.7 mm; B); hippocampus (-2.92 mm; C); SN and VTA (-4.92 mm; D). PRL, prelimbic cortex; $\mathrm{ACC}$, anterior cingulate cortex; NAcC, nucleus accumbens core; NAcS, nucleus accumbens shell; CPu, caudate putamen; M1, primary motor cortex; DG, dentate gyrus; SNpc, substantia nigra pars compacta; SNpr, substantia nigra pars reticulata; VTA, ventral tegmental area. The areas used for analysis of $\mathrm{TH}$-ir are highlighted in blue (CPu) and pink (NAc).

\section{MDA IMMUNOCYTOCHEMISTRY}

Sections through PRL/ACC, CPu/NAc/M1, hippocampus, and SN/VTA were examined for changes in MDA-adducted proteins. Briefly, slides were washed three times in PBS, and fixed in $4 \%$ paraformaldehyde $/ 0.9 \% \mathrm{NaCl}$ for $10 \mathrm{~min}$. The sections were then outlined with a PAP pen, followed by three washes in PBS and blocked with $10 \%$ normal horse serum in $0.3 \%$ Triton-X for $2 \mathrm{~h}$ at room temperature. The blocking solution was then removed, and the sections were incubated with a polyclonal rabbit anti-MDA antibody (1:200; Abcam, Cambridge, MA, USA), overnight at $4^{\circ} \mathrm{C}$ in humidified chambers. The sections were then washed with PBS, and incubated for $2 \mathrm{~h}$ at room temperature with a biotinylated goat anti-rabbit IgG antiserum (1:200; Vector Laboratories) diluted in $5 \%$ horse serum/PBS. Slides were then washed three times in PBS, incubated $1 \mathrm{~h}$ at room temperature in $\mathrm{ABC}$ solution (Elite ABC Kit, Vector Laboratories) and washed three more times in PBS. Bound antibody was detected by incubating the sections in a 3,3 -diaminobenzidine $/ \mathrm{Ni}^{+}$solution (Vector Laboratories) for 4-8 min. Slides were washed with deionized $\mathrm{H}_{2} \mathrm{O}$, dehydrated in a series of alcohols and coverslipped out of xylene.

\section{IMAGE ANALYSIS}

Slides from TH immunocytochemistry were analyzed using Image J (National Institutes of Health; http://rsb.info.nih.gov/ij), as previously described (Johnson-Davis et al., 2002) and the images captured with a video camera (CCD IEEE-1394, Scion Corporation, Frederick, MD, USA). Basic densitometric analysis yielded average density (gray) values over the region of interest. Before the measurement of sections, the linearity of the video camera to increasing signal intensity was determined using the average gray values of signals of known optical density from a photographic step tablet (Eastman Kodak Company, Rochester, NY, USA). The intensity of the light was adjusted such that the values measured from the slides of TH-ir brain sections fell within the linear portion of the system's response. Lighting and camera conditions remained constant during the process of capturing and collection of density measurements. Mean gray values were measured in the left hemisphere for the whole $\mathrm{CPu}$, which was designated as the area below the corpus callosum and above the anterior commissure, ending approximately at the ventral tip of the lateral ventricle (see Figure 1B, blue highlighted area). The whole NAc was designated as the area just dorsal to the anterior commissure, ending at the ventral tip of the lateral ventricle (in order to capture the entire core) and dorsal to the medial forebrain bundle/ventral pallidum on the ventral border (see Figure 1B, pink highlighted area). In order to correct for background labeling, the mean gray value of the white matter was subtracted from the mean gray value of the $\mathrm{CPu}$ or NAc, for each animal in the study.

For the analysis of MDA immunoreactivity (MDA-ir), sections were captured from a VistaVision microscope (VWR, Radnor, PA, USA) with a video camera (CCD Moticam 2300, Motic, Richmond, BC, Canada), using a $10 \times$ objective. Immunoreactivity was measured in the left hemisphere of each brain in the following pixel areas: $300 \times 500$ for PRL, $200 \times 200$ for ACC, $500 \times 300$ for M1, $400 \times 400$ for medial and lateral $\mathrm{CPu}, 200 \times 300$ for nucleus accumbens core, $150 \times 300$ for nucleus accumbens shell, $300 \times 100$ for CA1, $400 \times 100$ for CA3 and dentate gyrus (DG), a $500 \times 150$ oval for SNpc and substantia nigra pars reticulata (SNpr), and a circle with a diameter of 152 pixels for the VTA (Figure 1). The areas analyzed for semi-quantification were held constant for all experiments and was based on modified procedures from (Simpson et al., 1995; Choe et al., 2002; Horner et al., 2006). The number of MDA-labeled particles that exceeded the threshold density in each region of interest was determined using the particle analysis option in Image J. The pixel range for particle size was determined before analysis by outlining positively labeled cells from several randomly selected sections and determining the average size of the labeled cells in terms of pixel area. The lower limit for a "labeled cell" on the particle analysis setting was then set to the smallest number of pixels measured for any cell, whereas the upper limit was set at the maximal particle size on the particle analysis option on Image J. The threshold density was adjusted such that background staining was eliminated and the number of immunoreactive pixels per the selected area in each region of interest was measured above this threshold.

\section{STATISTICAL ANALYSIS}

The effect of multiple METH treatment on TH-ir and MDA-ir was analyzed using a two-tailed unpaired $t$-test for each region of interest. Differences in MDA-ir between the sub-regions of cortex or hippocampus following multiple METH treatment were analyzed using a one-way analysis of variance followed by Tukey's multiple comparisons post hoc test. Correlation between the number of MDA-ir particles and $\mathrm{TH}$-ir mean gray values for the $\mathrm{CPu}$ and NAc were calculated according to the Pearson method. The alpha level for all analyses was set at 0.05 . 


\section{RESULTS \\ EFFECTS OF MULTIPLE HIGH DOSES OF METH ON MDA-IR IN THE CPU AND NAC}

Exposure to multiple, high doses of METH resulted in an increase in MDA-ir particles in both the lateral and medial regions of $\mathrm{CPu}$ (Figure 2A). Semi-quantitative analysis revealed that multiple METH treatment significantly increased MDA-ir in the lateral $(t=5.03, p=0.0001)$ and medial $(t=5.04, p=0.0001) \mathrm{CPu}$ (Figure 2B). There was not a significant difference in the level of MDA-ir between the lateral and medial regions of $\mathrm{CPu}$ in METH-treated animals $(t=1.39, p=0.172)$. Exposure to multiple METH treatment also increased MDA-ir particles in the core and shell of NAc (Figure 3A). Semi-quantitative analysis showed that multiple METH treatment significantly increased MDA-ir in the core $(t=5.68, p<0.0001)$ and shell $(t=4.68$, $p=0.0004)$ of NAc (Figure 3B). There was not a significant difference between the level of MDA-ir induced by multiple METH treatment for the core vs. shell sub-regions of NAc $(t=2.04$, $p=0.06)$. However, multiple METH treatment induced significantly higher levels of MDA-ir in the $\mathrm{CPu}$ as compared to the NAc $(t=5.35, p<0.0001)$.

\section{EFFECTS OF MULTIPLE HIGH DOSES OF METH ON MDA-IR IN THE SN AND VTA}

Exposure to multiple, high doses of METH resulted in an increase in MDA-ir particles in the SNpc, but did not alter the amount of MDA-ir staining in the SNpr (Figure 4A). Exposure to multiple METH treatment also increased MDA-ir particles in the VTA (Figure 4A). Semi-quantitative analysis revealed that multiple METH treatment significantly increased MDA-ir in the SNpc $(t=6.62, p<0.0001)$, but not the SNpr $(t=0.65, p=0.949)$. Multiple METH treatment also significantly increased the number of MDA-ir particles in the VTA $(t=5.00, p=0.0002$; Figure $4 \mathrm{~B})$. Additional analysis revealed that multiple $\mathrm{METH}$ treatment resulted in a greater increase in MDA-ir in the SNpc as compared to the VTA $(t=11.17, p<0.0001)$.

\section{EFFECTS OF MULTIPLE HIGH DOSES OF METH ON MDA-IR IN THE CORTEX}

Exposure to multiple, high doses of METH resulted in an increase in MDA-ir particles all regions of cortex examined (Figure 5A). Semi-quantitative analysis revealed that multiple METH treatment significantly increased MDA-ir in the PRL $(t=5.29, p=0.0003)$, ACC $(t=7.20, p<0.0001)$, and M1 $(t=8.41, p<0.0001$; Figure 5B). Additional analysis revealed that the level of MDA-ir was

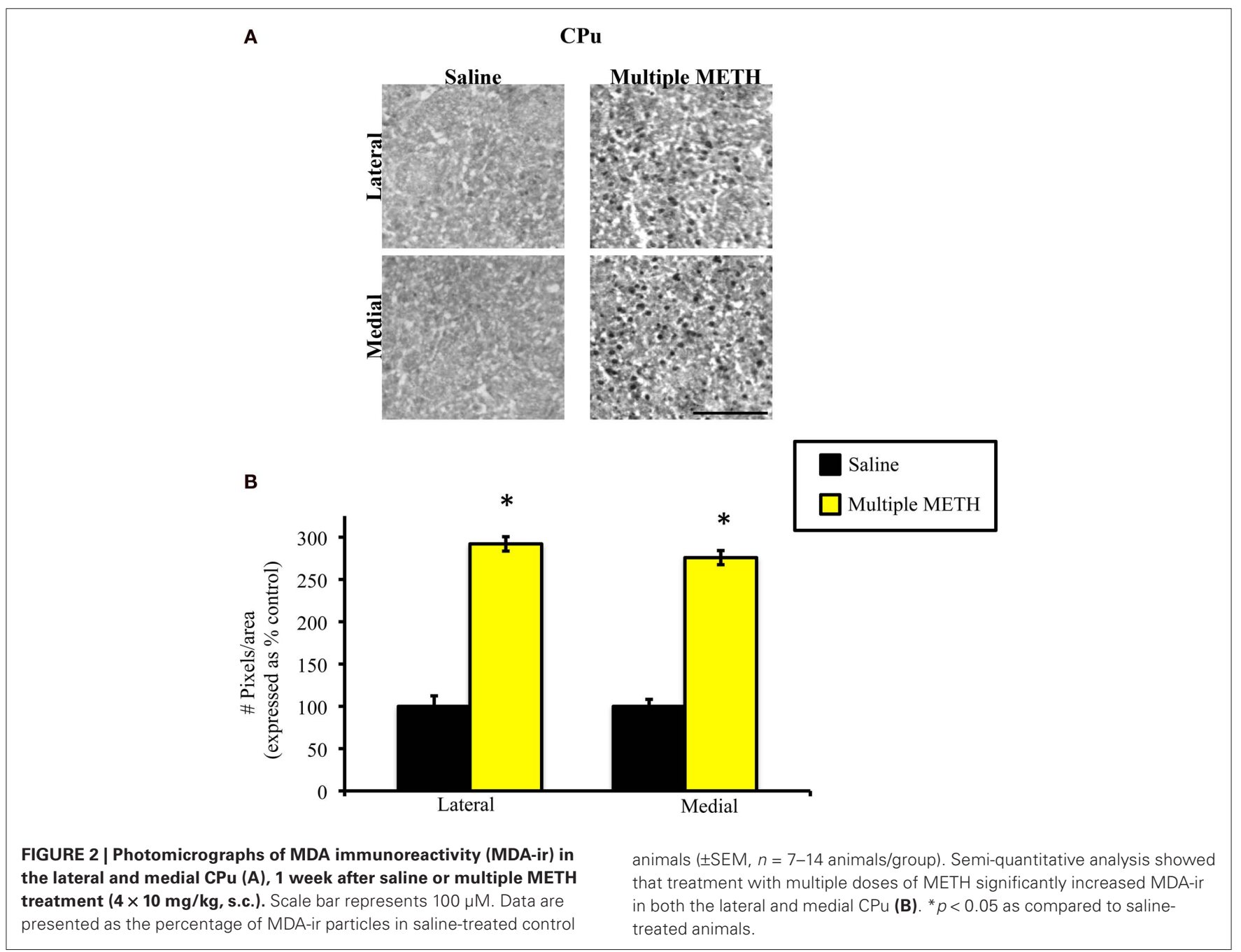


A

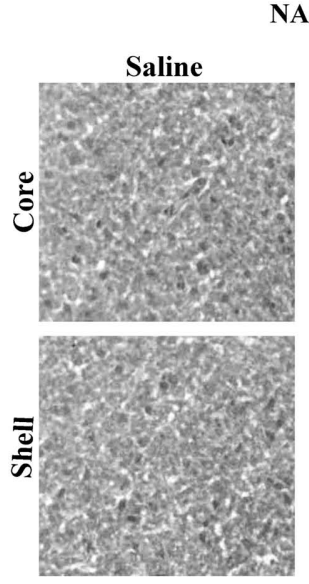

NAc

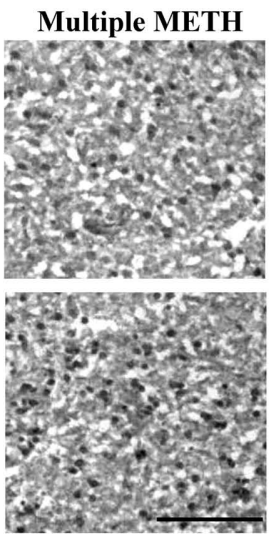

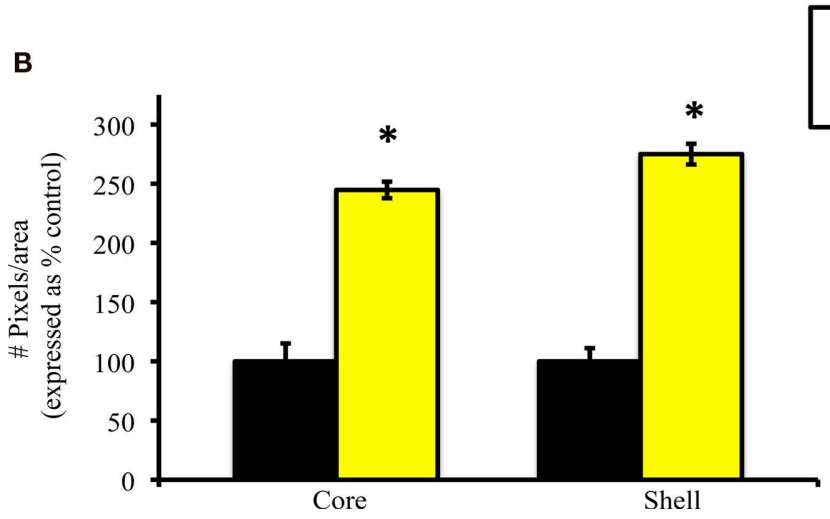

Saline

Multiple METH

FIGURE 3 | Photomicrographs of MDA-ir in the core and shell of the NAc (A), 1 week after saline or multiple METH treatment (4× 10 mg/kg, s.c.). Scale bar represents $100 \mu \mathrm{M}$. Data are presented as the percentage of MDA-ir particles in saline-treated control animals ( \pm SEM, $n=7-14$ animals/group). Semi-quantitative analysis showed that treatment with multiple doses of METH significantly increased MDA-ir in both the core and shell of the NAc (B). ${ }^{*} p<0.05$ as compared to saline-treated animals.

significantly greater in the ACC sub-region of cortex as compared to the PRL $(p<0.01)$ and M1 $(p<0.001)$ cortical sub-regions following multiple METH treatment.

\section{EFFECTS OF MULTIPLE HIGH DOSES OF METH ON MDA-IR IN THE HIPPOCAMPUS}

Exposure to multiple, high doses of METH resulted in an increase in MDA-ir particles in all regions of hippocampus examined (Figure 6A). Semi-quantitative analysis revealed that multiple METH treatment significantly increased MDA-ir in CA1 $(t=5.00$, $p=0.0001)$, CA3 $(t=6.46, p<0.0001)$, and DG $(t=4.01, p=0.002$; Figure 6B). Additional analysis revealed that the level of MDA-ir was significantly greater in the CA3 sub-region of hippocampus as compared to the CA1 $(p<0.001)$ and DG $(p<0.001)$ hippocampal sub-regions following multiple METH treatment.

\section{EFFECTS OF MULTIPLE HIGH DOSES OF METH ON TH-IR IN THE CPU AND NAC}

Treatment with multiple, high doses of METH decreased the amount of TH-ir in the $\mathrm{CPu}$, as well as the NAc (Figures 7A,B). Densitometric analysis revealed that exposure to multiple doses of METH significantly reduced staining for $\mathrm{TH}$ in the $\mathrm{CPu}(t=6.02$, $p<0.0001)$ and NAc $(t=2.98, p=0.009$; Figure 7C). However, the decrease in TH-ir induced by multiple METH treatment was significantly greater in the $\mathrm{CPu}$, as compared to the NAc $(t=6.25$, $p<0.0001)$, with $\mathrm{TH}$-ir being reduced to approximately 60 and $20 \%$ of control, respectively. In order to examine whether a relationship might exist between the degree of MDA-protein adducts induced by multiple METH treatment and the depletion of TH, we determined whether a correlation existed between the number of MDA-ir particles and the average gray values for $\mathrm{TH}$-ir. In the $\mathrm{CPu}$, a significant negative correlation existed between the number of MDA-ir particles (medial plus lateral regions) and the average gray values for TH-ir $(r=-0.82, p<0.0001$; Figure 7D). In the whole NAc, there was not a significant correlation between the number of MDA-ir particles (core plus shell regions) and the average gray values for TH-ir $(r=-0.40, p=0.10$; Figure $7 \mathrm{E})$. There was not a significant correlation between the number of MDA-ir particles and the average gray values for TH-ir when the core $(r=-0.40$, $p=0.15)$ and shell $(r=-0.47, p=0.09)$ were each analyzed separately (data not shown).

\section{DISCUSSION}

The purpose of the present study was to examine the effects of multiple high doses of METH on the modification of proteins by MDA in the nigrostriatal and mesoaccumbal systems, as well as 
A
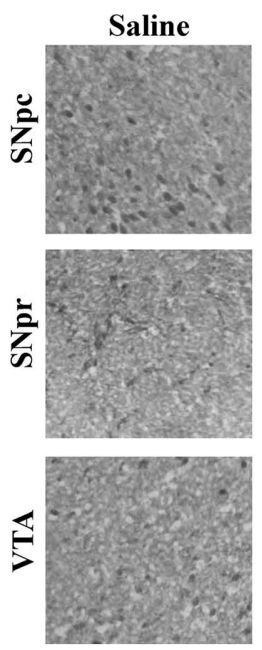

Multiple METH
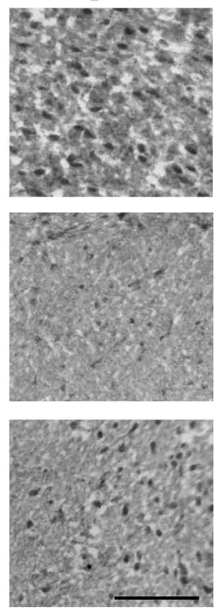

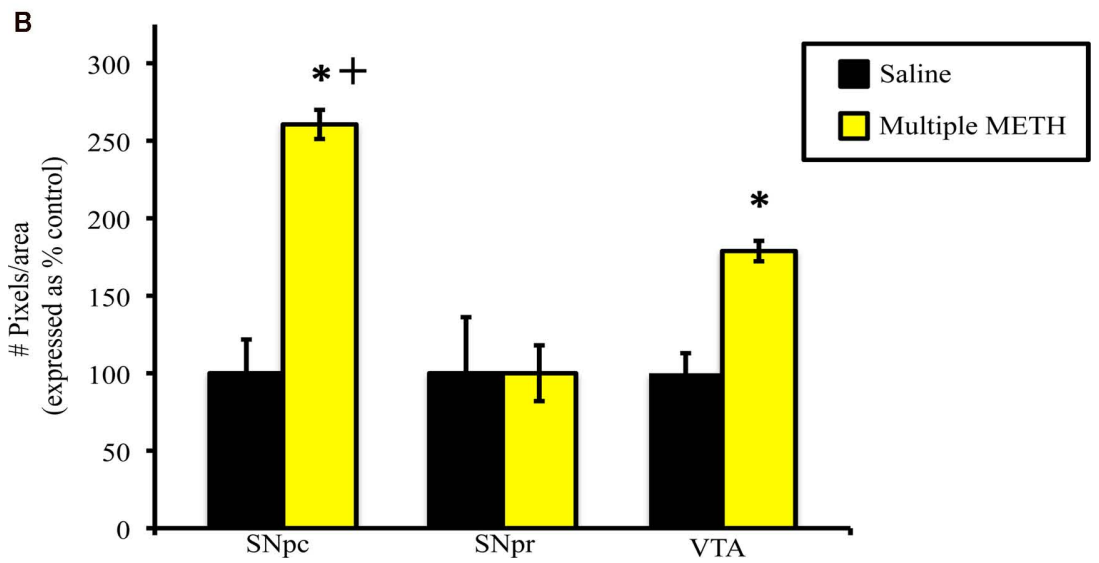

FIGURE 4 | Photomicrographs of MDA-ir in the SNpc, SNpr, and VTA (A) 1 week after saline or multiple METH treatment $(4 \times 10 \mathrm{mg} / \mathrm{kg}$, s.c.). Scale bar represents $100 \mu \mathrm{M}$. Data are presented as the percentage of MDA-ir particles in saline-treated control animals ( \pm SEM, $n=7-14$ animals/ group). Semi-quantitative analysis showed that treatment with multiple doses of METH significantly increased MDA-ir in the SNpc and VTA, but not in the SNpr (B). Multiple METH treatment also resulted in significantly greater MDA-ir in the SNpc, as compared to the VTA. ${ }^{*} p<0.05$ as compared to saline-treated animals. ${ }^{+} p<0.05$ as compared to the VTA in METHtreated animals. in cortex and hippocampus. We have shown that repeated METH administration results in increases in MDA-modified proteins in several regions of brain that persisted for 1 week after treatment, raising the possibility that long-term METH-induced neuronal damage could arise, in part, from the MDA-induced inactivation of essential protein functions. MDA-ir was significantly increased in the SNpc and CPu after multiple METH administration, which is in line with the toxic effects of multiple METH treatment on the dopaminergic neurons in this pathway. Multiple METH administration also significantly increased MDA-ir in the VTA and NAc, which was unexpected, as the dopaminergic neurons of the mesoaccumbal pathway are less severely affected by this treatment regimen. Furthermore, multiple METH treatment MDA-ir was significantly increased in the cortex and hippocampus, two regions that contain relatively lower levels of DA, indicating that MDA can induce significant protein adduction independent of the DA system. The data from the current study illustrate that there is widespread adduction of proteins by MDA following repeated treatment with
METH. Furthermore, our data show that MDA-protein adducts can occur in areas that receive less dense DA input, as well as in areas that are DA-dense and sensitive to the neurotoxic effects of METH treatment.

Our study focused on the accumulation of MDA-adducted proteins after exposure to a neurotoxic regimen of METH treatment as an indication of biomolecular damage by MDA that may impair cellular function. While we have yet to characterize the specific targets of MDA, it is important, nevertheless, to speculate about the potential mechanisms by which MDA production may contribute to METH-induced neurotoxicity, as these hypotheses will guide future research on METH-induced MDA neuronal damage. As mentioned above, MDA can induce cellular damage via DA-related mechanisms, or through the inhibition of mitochondrial function (Jinsmaa et al., 2009; Long et al., 2009). It is possible that in regions where there is a dense concentration of DA, such as the nigrostriatal pathway, DA-related mechanisms of MDA-induced damage may predominate and contribute to the destruction of 


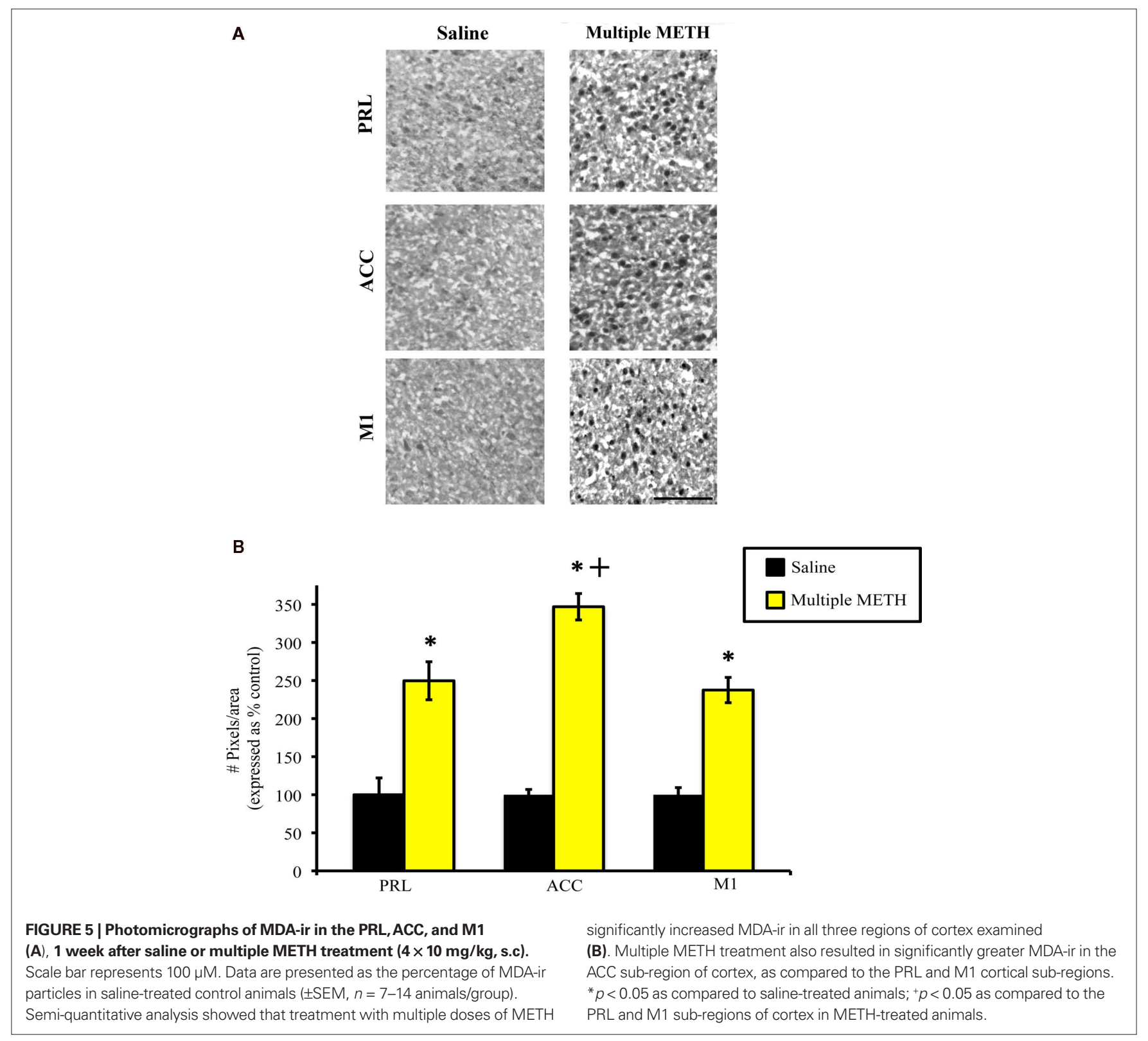

these neurons. DA is metabolized by monoamine oxidase (MAO) to produce 3,4-dihydroxyphenylacetaldehyde (DOPAL), which is then oxidized by aldehyde dehydrogenase-2 (ALDH-2) to produce 3,4-dihydroxyphenylacetic acid (DOPAC; Marchitti et al., 2007). Metabolism of DA by MAO and ALDH-2 prevents its cytoplasmic accumulation and its conversion to a neurotoxic quinone that conduces the eventual destruction of dopaminergic neurons (Lavoie and Hastings, 1999; Yamamoto and Bankson, 2005; Marchitti et al., 2007; Krasnova and Cadet, 2009). However, recent data indicates that MDA can lead to accumulation of DOPAL via irreversible inhibition of ALDH-2 activity (Jinsmaa et al., 2009). DOPAL is an aldehyde that is chemically reactive with proteins and has been shown to be several times more toxic to dopaminergic neurons than the DA-derived quinone (Rooke et al., 2000; Burke et al., 2003, 2004; Jinsmaa et al., 2009). In addition, the infusion of DOPAL into the SNpc or VTA results in higher levels of toxicity in the SNpc as compared to the VTA (Burke et al., 2003). Interestingly, the VTA has been shown to express higher levels of factors involved in cell survival and protection than the SNpc, suggesting that the SNpc may be more vulnerable to toxic insults than the VTA (Grimm et al., 2004). Thus, repeated exposure to METH may result in MDAmediated increases in DOPAL in both the SNpc and VTA, but the increase in DOPAL may have less of a toxic impact in the VTA, whereas the neurons of the SNpc may be more severely affected.

Interestingly, very little MDA-ir was found in the SNpr after multiple METH treatment, as compared to all other regions examined. The lack of MDA-ir induced by multiple METH treatment could be due to the fact that the SNpr is comprised of $\gamma$-aminobutyric acid (GABA)-containing neurons and also contains relatively low levels of DA (Gerfen and Bolam, 2010). However, somatodendritic release 


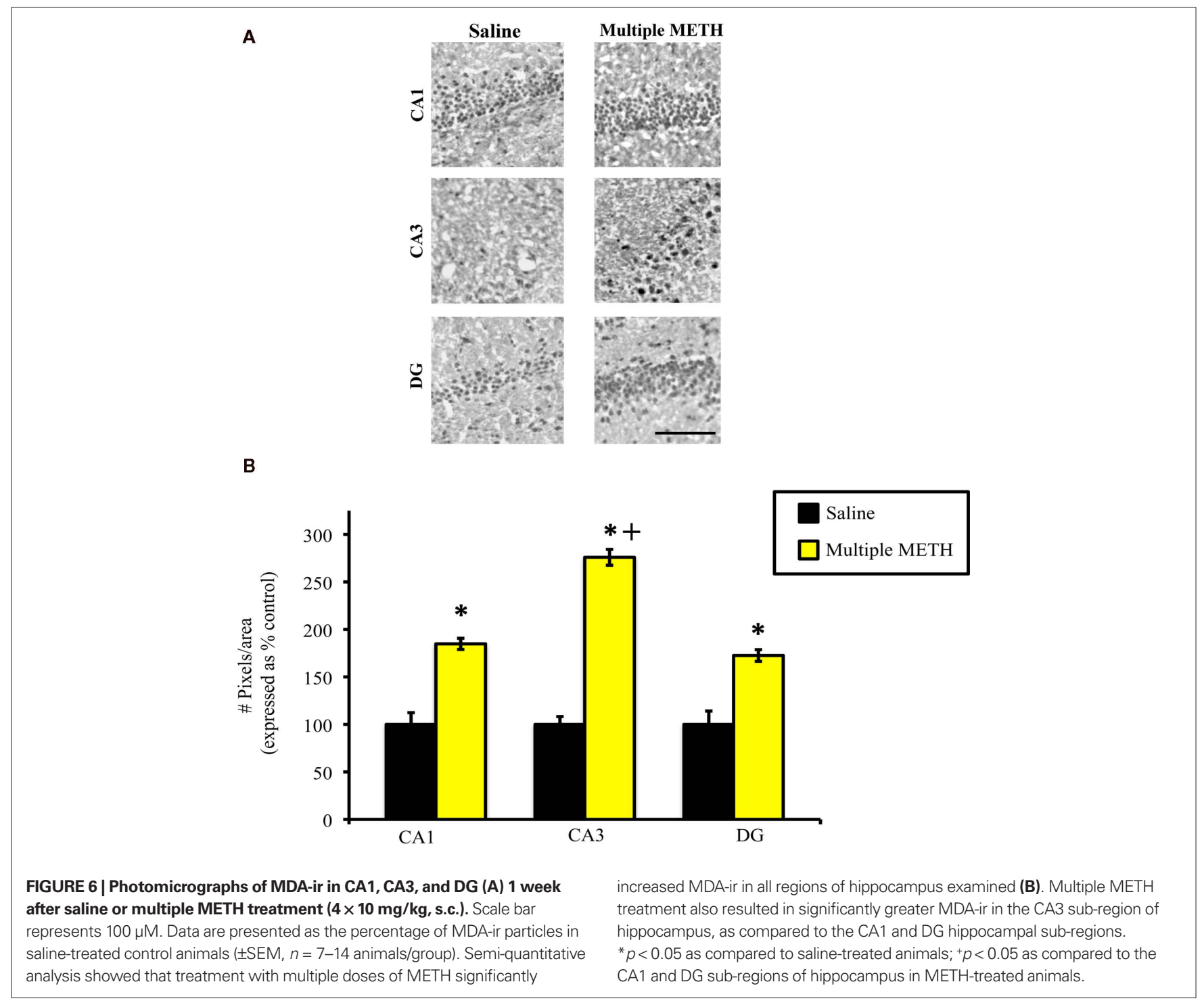

of DA into the SNpr from the dopaminergic neurons of the adjacent SNpc has been well-documented (Korf et al., 1976; Chéramy et al., 1981; Robertson et al., 1991; Heeringa and Abercrombie, 1995; Windels and Kiyatkin, 2006) and METH has been shown to increase extracellular levels of DA in the substantia nigra (Bustamante et al., 2002). Thus, DA levels may increase in the SNpr during METH treatment, setting the stage for DA-related increases in MDA-ir within this region, aside from any non-DA-related increases in MDA-ir that might also occur. At this point, however, it is not clear what factors might contribute to the relative resistance of the SNpr to the development METH-induced MDA-protein adducts following multiple METH treatment. Additional studies are needed in order to address the potential mechanisms that might underlie the resistance of the SNpr to the development of METH-induced MDA-protein adducts.

It is important to note, however, that our data indicate that repeated METH treatment results in significantly greater levels of MDA-ir in the nigrostriatal system vs. the mesoaccumbal system, raising the possibility that the enhanced neurotoxicity of the nigrostriatal system could simply be the result of relatively higher levels MDA-adducted proteins within this region. Interestingly, our data also show that there is a significant negative correlation between the loss of TH-ir and the increase in MDA-adducted proteins in the $\mathrm{CPu}$, indicating a relationship exists between increased MDA accumulation and depletion of TH in the nigrostriatal system. This inverse relationship between MDA-ir and TH-ir was not observed in the NAc. It is possible that in the nigrostriatal system, where TH levels decrease as levels of MDA-modified proteins increase, MDA-mediated inhibition of ALDH-2 and accumulation of DOPAL (which is structurally similar to DA) may lead to feedback inhibit on the production of $\mathrm{TH}$, due to cellular sensing of high DA-like compound levels (Burke et al., 2003). On the other hand, in the mesoaccumbal system, where TH levels do not appear to be related to levels of MDA-modified proteins, ALDH enzymes may be able to effectively eliminate DOPAL and avoid feedback inhibition of TH synthesis. Indeed, other ALDH isoforms, such as ALDH-1, can compensate for DOPAL oxidation in the event of ALDH-2 blockade (Manzer et al., 2003; Marchitti et al., 2007; Jinsmaa et al., 


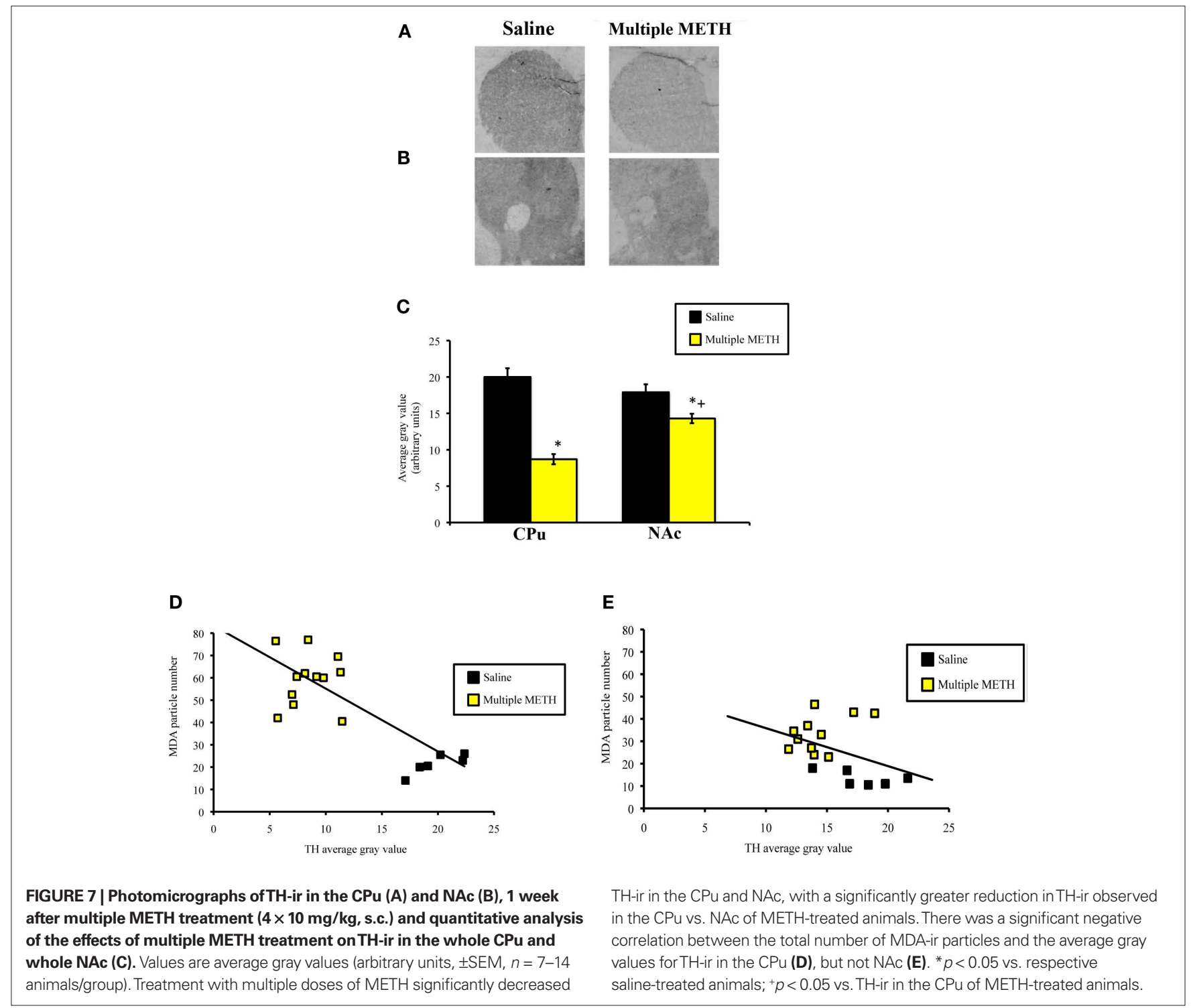

2009). It is possible that in the mesoaccumbal system, inhibition of ALDH-2 by MDA may be ameliorated by ALDH-1-mediated oxidation of DOPAL to DOPAC. However, it is important to point out that ALDH-1 is expressed to a higher degree in the SNpc as compared to the VTA (McCaffery and Drager, 1994; Jinsmaa et al., 2009). Clearly, additional studies are needed to further examine the relative contribution of ALDH isoforms to the clearance of MDA-mediated increases in DOPAL within the SNpc and VTA.

Multiple METH treatment also increased the levels of MDAmodified proteins in the prefrontal cortex, as MDA-ir was increased in both the PRL and ACC. The frontal cortex contains relatively lower levels of $\mathrm{DA}$ than the $\mathrm{CPu}$, but exposure to multiple high doses of METH has been shown to result in depletion of DA levels and TH hydroxylase activity in the frontal cortex (Hotchkiss and Gibb, 1980; Ricaurte et al., 1980). Therefore, it is possible that increased levels of MDA in the frontal cortex contribute to the loss of DA levels and markers in this region, through the accumulation of toxic DA metabolites as a result of the inhibition of ALDH-2, as described above. However, the increases in MDA-adducted proteins observed in the sub-regions of prefrontal cortex after multiple METH treatment were similar in magnitude to what was observed in the $\mathrm{CPu}$ (data not shown), despite the relative differences in DA levels between these two regions, suggesting that MDA-protein modifications may occur in non-DA neurons, leading to widespread neuronal damage. In support of this observation is the finding that multiple METH treatment can induce apoptosis in non-DA neurons in the cortex (Pu et al., 1996; Cadet et al., 2003). Thus, following multiple METH treatment, MDA may also lead to damage of non-DA neurons in the cortex via direct adduction of proteins and nucleic acids or disruption of mitochondrial function. This non-DA-related mechanism of cellular damage could be responsible for a relatively larger portion of $\mathrm{METH}$-induced neuronal damage than the DA-mediated mechanism of neuronal damage, given the lower levels of DA input in the cortex. Future 
studies examining which proteins are specifically adducted by MDA within the cortex, and their relative contribution to the depletion of DA markers and apoptosis of non-DA neurons in the cortex will shed light on this issue.

Our data also indicate that multiple high doses of METH increase MDA-modified proteins in the hippocampus, as MDA-ir was significantly increased in the CA1, CA3, and DG. The hippocampus receives sparse input from DA afferents, but receives dense inputs from serotonergic afferents (Rau et al., 2006). Thus, it is likely in this region, the toxic effects of MDA adduction have little to do with the DA system and inhibition of ALDH-2 activity. Treatment with multiple high doses of METH depletes serotonin (5-HT) concentrations in the hippocampus, as well as the rate-limiting enzyme in 5-HT synthesis, tryptophan hydroxylase (TPH; Hotchkiss and Gibb, 1980; Ricaurte et al., 1980, 1983; Bakhit et al., 1981; Green et al., 1992). In this instance, it is possible that MDA contributes to METH-induced damage of 5-HT neurons through direct modification of proteins essential for the function and survival of these neurons. Multiple METH treatment also results in severe depletion of 5-HT and TPH in the CPu and frontal cortex (Hotchkiss and Gibb, 1980; Ricaurte et al., 1980), and it is possible that direct MDA adduction of proteins necessary for the viability of serotonergic input to these regions contribute to this phenomenon. Alternatively, MDA-induced ROS generation could lead to oxidation of 5-HT, producing the metabolite tryptamine-4,5-dione, which may contribute to serotonergic neurotoxicity following METH treatment (Wrona and Dryhurst, 2001). However, a relationship between MDA-induced ROS generation and production of 5-HT-derived toxic metabolites has not been established. Additional studies are needed to investigate the potential role that MDA accumulation might play in the destruction of 5-HT neurons by METH.

Interestingly, there was a greater degree of MDA-ir induced by multiple METH treatment in the CA3 sub-region, as compared to the CA1 and DG sub-regions of hippocampus and a greater degree of MDA-ir induced by multiple METH in the ACC, as compared to

\section{REFERENCES}

Acikgoz, O., Gonenc, S., Kayatekin, B. M., Uysal, N., Pekcetin, C., Semin, I., and Gure, A. (1998). Methamphetamine causes lipid peroxidation and an increase in superoxide dismutase activity in the rat striatum. Brain Res. 813, 200-202.

Aldini, G., Dalle-Donne, I., Facino, R. M., Milzani, A., and Carini, M. (2007). Intervention strategies to inhibit protein carboxylation by lipoxidationderived reactive carbonyls. Med. Res. Rev. 27, 817-868.

Bakhit, C., Morgan, M.E., Peat, M.A., and Gibb, J.W. (1981). Long-term effects of methamphetamine on synthesis and metabolism of 5-hydroxytryptamine in various regions of the rat brain. Neuropharmacology 20, 1135-1140.

Baxter, L. R. Jr., Schwartz, J. M., Phelps, M. E., Mazziotta, J. C., Guze, B. H., Selin, C. E., Gerner, R. H., and Sumida, R. M. (1989). Reduction of prefrontal cortex glucose metabolism common to three types of depression. Arch. Gen. Psychiatry 46, 243-250.

Brown, J. M., Quinton, M. S., and Yamamoto, B. K. (2005). Methamphetamine-induced inhibiroles of glutamate and peroxynitrate. J. Neurochem. 95, 429-436.

Brown, J. M., and Yamamoto, B. K. (2003). Effects of amphetamine on mitochondrial function: role of free radicals and oxidative stress. Pharmacol. Ther. 99, 45-53.

Burke, W. J., Li, S. W., Chung, H. D., Ruggiero, D. A., Kristal, B. S., Johnson, E. M., Lampe, P., Kumar, V. B., Franko, M., Williams, E. A., and Zahm, D. S. (2004). Neurotoxicity of MAO metabolites of catecholamine neurotransmitters: role in neurodegenerative diseases. Neurotoxicology 25, 101-115.

Burke, W. J., Li, S. W., Williams, E. A., Nonneman, R., and Zahm, D.S. (2003). 3,4-Dihydroxyphenylacetaldehyde is the toxic dopamine metabolite in vivo: tion of mitochondrial complex II:

the PRL and M1 sub-regions of cortex. It is unclear what potential mechanism(s) might underlie the enhanced induction of MDA-ir in the CA3 sub-region of hippocampus or ACC sub-region of cortex. However, damage to the CA3 could explain the deficits in short-term memory and spatial learning that has been observed in human METH abusers and in animals exposed to repeated, high doses of METH (McEwen, 1999; Chapman et al., 2001; Thompson et al., 2004), while damage to the ACC could explain the deficits in cognition and emotional processing often observed in METH addicts (Baxter et al., 1989; Clow and Hammer, 1991; Bush et al., 2000). Clearly, additional studies are needed in order to determine the factors that might underlie the relative enhancement of METHinduced MDA-protein adducts within these sub-regions of cortex and hippocampus.

In summary, treatment with multiple high doses of METH increases MDA-adducted proteins in the nigrostriatal system, which is the system that is the most vulnerable to the neurotoxic effects this treatment regimen. Multiple METH treatment also increased levels of MDA-modified proteins in the mesoaccumbal system, yet this system suffers comparatively less neurotoxic damage than the nigrostriatal system. It is possible that the neurotoxic damage to the nigrostriatal system by multiple METH treatment is due to inhibition of ALDH-2 by MDA, resulting in accumulation of DOPAL. It is possible that MDA also inhibits ALDH-2 activity in the mesoaccumbal system, but due to relative differences in ALDH enzymes and cell survival molecules expressed in this system, the neurotoxic effects of multiple METH treatment may be less deleterious. Studies are currently underway in our laboratory to determine whether multiple METH treatment alters ALDH-2 expression and levels of DOPAL in the nigrostriatal vs. mesoaccumbal systems. In addition, multiple METH treatment also increased MDA-modified proteins in the cortex and hippocampus, two regions that are less densely innervated by DA afferents, but nonetheless suffer significant damage as a result of METH treatment, suggesting that MDA may directly contribute to the damage of non-DA neurons within these regions.

implications for Parkinson's disease pathogenesis. Brain Res. 989, 205-213. Bush, G., Luu, P., and Posner, M. I. (2000). Cognitive and emotional influences in anterior cingulate cortex. Trends Cogn. Sci. 4, 215-222.

Bustamante, D., You, Z. B., Castel, M. N., Johansson, S., Goiny, M., Terenius, L., Hokfelt, T., and Herrera-Marschitz, M. (2002). Effect of single and repeated methamphetamine treatment on neurotransmitter release in substantia nigra and neostriatum of the rat. J. Neurochem. 83, 645-654.

Cadet, J. L., Subramaniam, J., and Deng, X. (2003).Speed kills: cellular and molecularbases of methamphetamine-induced nerveterminal degeneration and neuronal apoptosis. FASEB J. 17, 1775-1788.

Chapman, D. E., Hanson, G. R., Kessner, R. P., and Keefe, K. A. (2001). Longterm changes in basal ganglia function after a neurotoxic regimen of methamphetamine. J. Pharmacol. Exp. Ther. 296, 520-527.
Chéramy, A., Leviel, V., and Glowinski, J. (1981). Dendritic release of dopamine in the substantia nigra. Nature 289 , 537-542.

Choe,E.S., Chung, K. T., Mao, L., and Wang, J. Q. (2002). Amphetamine increases phosphorylation of extracellular signal-regulated kinase and transcription factors in the rat striatum via group I metabotropic glutamate receptors. Neuropsychopharmacology27,565-575. Clow, D. W., and Hammer, R. P. Jr. (1991). Cocaine abstinence following chronic treatment alters cerebral metabolism in dopaminergic reward regions; bromocriptine enhances recovery. Neuropsychopharmacology 4, 71-75.

Del Rio, D., Stewart, A. J., and Pellegrini, N. (2005). A review of recent studies on malondialdehyde as a toxic molecule and biological marker of oxidative stress. Nutr. Metab. Cardiovasc. Res. 15, 316-328.

Emson, P. C., Koob, G., and Iversen, L. L. (1977). Origin of dopaminergic affer- 
ents to the rat frontal cortex [proceedings]. Br. J. Pharmacol. 60, 270P-271P.

Fitzmaurice, P. S., Tong, J., Yazdanpanah, M., Liu, P. P., Kalasinsky, K. S., and Kish, S. J. (2006). Levels of 4-hydroxynonenal and malondialdehyde are increased in brain of human chronic users of methamphetamine. J. Pharmacol. Exp. Ther. 319, 703-709.

Gerfen, C. R., and Bolam, J.P. (2010). “The neuroanatomical organization of the basal ganglia," in Handbook of Basal Ganglia Structure and Function, eds H. Steiner and K. Y. Tseng (New York, NY: Academic Press), 3-23.

Gluck, M. R., Moy, L. Y., Jayatilleke, E., Hogan, K. A., Manzino, L., and Sonsalla, P.K. (2001). Parallel increases in lipid and protein oxidative markers in several mouse brain regions after methamphetamine treatment. J. Neurochem. 79, 152-160.

Green, A. R., De Souza, R. J., Williams, J.L., Murray, T. K., and Cross, A. J. (1992). The neurotoxic effects of methamphetamine on 5-hydroxytryptamine and dopamine in brain: evidence for the protective effect of chlormethiazole. Neuropharmacology31,315-321.

Grimm, J., Mueller, A., Hefti, F., and Rosenthal,A. (2004). Molecular basisfor catecholaminergic neuron diversity. Proc. Natl. Acad. Sci. U.S.A. 101,13891-13896.

Heeringa, M. J., and Abercrombie, E. D. (1995). Biochemistry of somatodendritic dopamine release in substantia nigra: an in vivo comparison with striatal dopamine release. J. Neurochem. 65, 192-200.

Horner, K. A., Gilbert, Y. E., and Cline, S. D. (2010). "Multiple high doses of methamphetamine increase malondialdehyde immunoreactivity in the nigrostriatal and mesolimbic systems of rat brain," in Paper Presented at the Triennial International Basal Ganglia Society Meeting, Long Branch.

Horner, K. A., Westwood, S. C., Hanson, G. R., and Keefe, K. A. (2006). Multiple high doses of methamphetamine increase the number of preproneuropeptideY mRNA-expressing neurons in the striatum of rat via a dopamine D1 receptor-dependent mechanism. J. Pharmacol. Exp. Ther. 319, 414-421.

Hotchkiss, A. J., and Gibb, J. W. (1980). Long-term effects of multiple doses of methamphetamine on tryptophan hydroxylase and tyrosine hydroxylase activity in rat brain. J. Pharmacol. Exp. Ther. 214, 257-262.

Jayanthi, S., Ladenheim, B., and Cadet, J.L. (1998). Methamphetamine-induced changes in antioxidant enzymes and lipid peroxidation in copper/zincsuperoxide dismutase transgenic mice. Ann. N. Y. Acad. Sci. 844, 92-102.

Jinsmaa, Y., Florang, V. R., Rees, J. N., Anderson, D. G., Strack, S., and
Doorn, J. A. (2009). Products of oxidative stress inhibit aldehyde oxidation and reduction pathways in dopamine catabolism yielding elevated levels of a reactive intermediate. Chem. Res. Toxicol. 22, 835-841.

Johnson-Davis, K. L., Hanson, G. R., and Keefe, K. A. (2002). Long-term post-synaptic consequences of methamphetamine on preprotachykinin mRNA expression. J. Neurochem. 82, 1472-1479.

Kim, H.-C., Jhoo, W.-K., Choi, D.-Y., Im, D.-H., Shin, E.-J., Suh, J.-H., Floyd, R. A., and Bing, G. (1999). Protection of methamphetamine nigrostriatal toxicity by dietary selenium. Brain Res. 851, 76-86.

Kita,T.,Shimada,K.,Mastunari,Y.,Wagner, G. C., Kubo, K., and Nakashima, T. (2000). Methamphetamine-induced striatal dopamine neurotoxicity and cyclooxygenase-2 protein expression in BALB/c mice. Neuropharmacology 39, 399-406.

Kogan, F. J., Nichols, W. K., and Gibb, J. W. (1976). Influence of methamphetamine on nigral and striatal tyrosine hydroxylase activity and on striatal dopamine levels. Eur. J. Pharmacol. 36, 363-371.

Korf, J., Zieleman, M., and Westerlnk, B. H. C. (1976). Dopamine release in the substantia nigra? Nature 260,257-258.

Krasnova, I. N., and Cadet, J. L. (2009). Methamphetamine toxicity and the messengers of death. Brain Res. Rev. 60, 397-407.

Lavoie, M. J., and Hastings, T. G. (1999). Dopamine quinone formation and protein modification associated with the striatal neurotoxicity of methamphetamine: evidence against a role for extracellular dopamine. J. Neurosci. 19, 1484-1491.

Lieberman, M., and Marks, A. D. (2009). Marks' Basic Medical Biochemistry: A Clinical Approach, Baltimore, MD: Lippincott Williams \& Wilkins.

Long, J., Liu, C., Sun, L., Gao, H., and Liu, J. (2009). Neuronal mitochondrial toxicity of malondialdehyde: inhibitory effects on respiratory function and enzyme activities in rat brain mitochondria. Neurochem. Res. 34 , 786-794.

Manzer, R., Qamar, L., Estey, T., Pappa, A., Petersen, D. R., and Vasiliou, V. (2003). Molecular cloning and baculovirus expression of the rabbit corneal aldehyde dehydrogenase (ALDH1A1) cDNA. DNA Cell Biol. 22, 329-338.

Marchitti,S.A.,Deitrich,R.A., and Vasiliou, V. (2007). Neurotoxicity and metabolism of the catecholamine-derived 3,4-dihydroxyphenylacetaldehyde, a reactive intermediate of dopamine metabolism by 4-hydroxy-2-nonenal. Pharmacol. Rev. 59, 125-150.
Maxwell, J. C. (2005). Emerging research on methamphetamine. Curr. Opin. Psychiatry 18, 235-242.

McCaffery, P., and Drager, U. C. (1994). High levels of a retinoic acidgenerating dehydrogenase in the mesotelencephalic dopamine system. Proc. Natl. Acad. Sci. U.S.A. 91, 7772-7776.

McEwen, B. (1999). Stress and hippocampal plasticity. Annu. Rev. Neurosci. 22, 105-122.

Moore, K., and Roberts, L. J. II. (1998). Measurement of lipid peroxidation. Free Radic. Res. 28, 659-671.

$\mathrm{Pu}, \mathrm{C}$., Broening, H. W., and Vorhees, C. V. (1996). Effect of methamphetamine on glutamate-positive neurons in the adult and developing rat somatosensory cortex. Synapse 23, 328-334.

Rau, K. S., Birdsall, E., Volz, T. J., Riordan, J. A., Baucum, A. J. I., Adair, B. P., Bitter, R., Gibb, J. W., Hanson, G. R., and Fleckenstein, A. E. (2006). Methamphetamine administration reduces hippocampal vesicular monoamine transporter-2 uptake. $J$. Pharmacol. Exp. Ther. 318, 676-682.

Ricaurte, G. A., Fuller, R. W., Perry, K. W., Seiden, L. S., and Schuster, C. R. (1983). Fluoxetine increases longlasting neostriatal dopamine depletion after administration of d-methamphetamine and d-amphetamine. Neuropharmacology 22, 1165-1169.

Ricaurte, G.A., Schuster, C. R., and Seiden, L. S. (1980). Long-term effects of repeated methylamphetamine administration on dopamine and serotonin neurons in the rat brain: a regional study. Brain Res. 193, 153-163.

Robertson, G. S., Damsma, G., and Fibiger, H. C. (1991). Characterization of dopamine release in the substantia nigra by in vivo microdialysis in freely moving rats. J. Neurosci. 11,2209-2216.

Rooke, N., Li, D.-J., Li, J., and Keung, W.M. (2000). The mitochondrial monoamine oxidase-aldehyde dehydrogenase pathway: a potential site of action of daidzin. J. Med. Chem. 43, 4169-4179.

Scatton, B., Javoy-Agid, F., Rouquier, L., Dubois, B., and Agid, Y. (1983). Reduction of cortical dopamine, noradrenaline, serotonin and their metabolites in Parkinson's disease. Brain Res. 275, 321-328.

Seiden, L. S., and Ricuarte, G. A. (1987). "Neurotoxicity of methamphetamine and related drugs," in Psychopharmacology: The Third Generation of Progress, ed. H. Meltzer (New York: Raven Press), 359-366.

Simpson, J.N., Wang, J.Q., and McGinty, J.F. (1995). Repeated amphetamine administration induces a prolonged augmentation of phosphorylated cyclase response element-binding protein and fos-related antigen immunoreactivity in rat striatum. Neuroscience 69, 441-457.
Sternberger, L. (1979). The unlabeled antibody (PAP) method, introduction. J. Histochem. Cytochem. 27, 1657.

Substance Abuse and Mental Health Services Administration, Office of Applied Studies. (2010). The DAWN Report: Emergency Department Visits Involving Methamphetamine: 2004 to 2008. Rockville, MD: Drug Abuse Warning Network.

Thompson, P. M., Hayashi, K. M., Simon, S. L., Geaga, J. A., Hong, M. S., Sui, Y., Lee, J. Y., Toga, A. W., Ling, W., and London, E. D. (2004). Structural abnormalities in the brains of human subjects who use methamphetamine. J. Neurosci. 24, 6028-6036.

Volkow, N. D., Chang, L., Wang, G. J., Fowler, J. S., Franscechi, D., Sedler, M. J., Gatley, S. J., Hintzemann, R., Ding, Y. S., and Wong, C. (2001). Higher cortical and lower subcortical metabolism in detoxified methamphetamine abusers. Am. J. Psychiatry 158, 383-389.

Windels, F., and Kiyatkin, E. A. (2006). Dopamine action in the substantia pars reticulata: iontophoretic studies in awake, unrestrained rats. Eur. J. Neurosci. 24, 1385-1394.

Wrona, M. A., and Dryhurst, G. (2001). A putative metabolite of serotonin, tryptamine-4,5-dione, is an irreversible inhibitor of tryptophan hydroxylase: possible relevance to the serotonergic neurotoxicity of methamphetamine. Chem. Res. Toxicol. 14, 1184-1192.

Yamamoto, B. K., and Bankson, M. G. (2005). Amphetamine neurotoxicity: cause and consequence of oxidative stress. Crit. Rev. Neurobiol. 17, 87-117.

Yamamoto, B. K., and Zhu, W. (1998). The effects of methamphetamine on the production of free radicals and oxidative stress. J. Pharmacol. Exp. Ther. 287, 107-114.

Conflict of Interest Statement: The authors declare that the research was conducted in the absence of any commercial or financial relationships that could be construed as a potential conflict of interest.

Received: 21 December 2010; accepted: 27 April 2011; published online: 09May 2011. Citation: Horner KA, Gilbert YE and Cline SD (2011) Widespread increases in malondialdehyde immunoreactivity in dopamine-rich and dopamine-poor regions of rat brain following multiple, high doses of methamphetamine. Front. Syst. Neurosci. 5:27. doi: 10.3389/fnsys.2011.00027

Copyright $\odot 2011$ Horner, Gilbert and Cline. This is an open-access article subject to a non-exclusive license between the authors and Frontiers Media SA, which permits use, distribution and reproduction in other forums, provided the original authors and source are credited and other Frontiers conditions are complied with. 